\title{
Indicators of Physical Activity Among Children and Youth in 9 Countries With Low to Medium Human Development Indices: A Global Matrix 3.0 Paper
}

\author{
Taru Manyanga, Joel D. Barnes, Chalchisa Abdeta, Ade F. Adeniyi, Jasmin Bhawra, \\ Catherine E. Draper, Tarun R. Katapally, Asaduzzaman Khan, Estelle Lambert, \\ Daga Makaza, Vida K. Nyawornota, Reginald Ocansey, Narayan Subedi, \\ Riaz Uddin, Dawn Tladi, and Mark S. Tremblay
}

\begin{abstract}
Background: This study compares results of physical activity report cards from 9 countries with low to medium human development indices, participating in the Global Matrix 3.0 initiative. Methods: Country-specific report cards were informed by relevant data and government policy documents, reporting on 10 core indicators of physical activity for children and youth. Data were synthesized by report card working groups following a harmonized process. Grade assignments for each indicator utilized a standard grading rubric. Indicators were grouped into one of 2 categories: daily behaviors and settings and sources of influence. Descriptive statistics (average grades) were computed after letter grades were converted into interval variables. Spearman's rank correlation coefficients were calculated for all correlation analyses. Results: Mean grades for daily behaviors were higher (C) than those for settings and sources of influence (D+). Twenty-nine out of the possible 90 grades were assigned an incomplete. There were moderate to strong positive and negative relationships between different global indices and overall physical activity, organized sport and physical activity, active play, family, community and environment, and government. Conclusions: Findings demonstrate an urgent need for high-quality data at the country level in order to better characterize the physical activity levels of children and youth in countries with low to medium human development indices.
\end{abstract}

Keywords: active living, policy, daily physical activity behaviors, settings and sources of influence

Evidence from studies conducted mainly in countries with high or very high human development indices (HDIs) show that regular physical activity among children and youth is associated with physical, psychosocial, and cognitive well-being, ${ }^{1}$ decreased adiposity, ${ }^{2,3}$ improved academic achievement, ${ }^{1,4}$ and brain health

\footnotetext{
Manyanga, Barnes, and Tremblay are with Healthy Active Living and Obesity Research Group, Children's Hospital of Eastern Ontario Research Institute, Ottawa, Ontario, Canada. Abdeta is with Hiwot Fana Specialized Haramaya University Hospital, Harar, Ethiopia. Adeniyi is with the College of Medicine, University of Ibadan, Ibadan, Nigeria; and Nigerian Heart Foundation, Ibadan and Lagos, Nigeria. Bhawra is with the School of Public Health and Health Systems, University of Waterloo, Waterloo, Ontario, Canada. Draper is with the University of the Witwatersrand, Johannesburg, South Africa; and the University of Cape Town, Cape Town, South Africa. Katapally is with the Johnson Shoyama Graduate School of Public Policy, University of Regina, Regina, Saskatchewan, Canada; and the Department of Community Health and Epidemiology, College of Medicine, University of Saskatchewan, Saskatoon, Saskatchewan, Canada. Khan and Uddin are with the School of Health and Rehabilitation Sciences, University of Queensland, St Lucia, Queensland, Australia. Lambert is with the University of Cape Town Research Centre for Health through Physical Activity, Lifestyle and Sport, Department of Human Biology, Faculty of Health Sciences, University of Cape Town, Cape Town, South Africa. Makaza is with the National University of Science and Technology, Bulawayo, Zimbabwe. Nyawornota is with the School of Education and Leadership, University of Ghana, Accra, Ghana. Ocansey is with the University of Ghana, Accra, Ghana. Subedi is with Maharajgunj Medical Campus, Institute of Medicine, Tribhuvan University, Maharajgunj, Nepal. Uddin is also with the Department of Pharmacy, Stamford University Bangladesh, Dhaka, Bangladesh. Tladi is with the Department of Physical Education, Health and Recreation, University of Botswana, Gaborone, Botswana. Manyanga (tmanyanga@cheo.on. ca) is corresponding author.
}

and development. ${ }^{5}$ Despite these known benefits, a significant proportion of children and adolescents do not accumulate the recommended amount of daily physical activity. ${ }^{6,7}$ Rhodes et $\mathrm{al}^{6}$ reported that 4 out of 5 adolescents between the ages of 11 and 17 did not meet the recommended daily minimum of 60 minutes of moderate- to vigorous-intensity physical activity. Furthermore, both insufficient physical activity and sedentary behaviors ${ }^{8}$ among children and youth have been increasing globally. ${ }^{7,9}$

Sedentary behaviors ${ }^{10-12}$ and insufficient physical activity ${ }^{2,3}$ in children and youth pose a significant public health challenge, because these behaviors are independently associated with noncommunicable diseases in adulthood. ${ }^{13}$ In addition, insufficient physical activity is currently the fourth leading risk factor for mortality globally. ${ }^{14}$ Many countries with low to medium HDIs are undergoing industrialization and economic growth along with rural to urban migration; these factors have led to the unintended consequences of rapid urbanization and densely populated cities. The United Nations estimates that within the next few decades, the largest and fastest growth in urban populations will be in Africa and Asia, places that currently remain mostly rural..$^{15}$ High population density in cities, particularly in low-income neighborhoods, often reduces walkability. ${ }^{16}$ In addition, urbanization itself, ${ }^{17}$ and the accompanying increase in motorized transportation ${ }^{7}$ use in densely populated cities, ${ }^{18}$ may decrease the amount of daily utilitarian or habitual physical activity. ${ }^{8}$

Promoting physical activity and reducing both insufficient physical activity and sedentary behaviors are important for promoting child health. In the context of the ongoing physical activity transition (the behavioral shift from traditionally active to more industrialized and sedentary lifestyles), ${ }^{8,14,19,20}$ global strategies 
(eg, sustainable development goals ${ }^{21}$ and the World Health Organization global action plan on physical activity 2018-2030 ${ }^{22}$ ) that identify specific indicators of progress and global interventions, including strategies for countries at different levels of human development that have unique contexts, cultures, challenges, and opportunities, may be needed to prevent accelerated physical activity transitions. $^{23}$

To better understand and develop global strategies for curbing insufficient physical activity among children and youth, reducing sedentary behaviors, and encouraging more active lifestyles, the Active Healthy Kids Global Alliance (AHKGA: www.active healthykids.org) has advocated for and developed a harmonized approach to data synthesis. ${ }^{23-25}$ This initiative facilitates the development of report cards ${ }^{26}$ on indicators of child and youth physical activity in countries that participate in the global matrix initiatives. ${ }^{23,24}$ However, documented variation in physical activity and other lifestyle behaviors across regions and cultures ${ }^{23,24}$ and a lack of data regarding the extent of or levels of inequalities in and determinants of movement behaviors among children and youth $^{27}$ in many countries with low to medium HDIs continue to make it challenging to draw meaningful global comparisons or conclusions.

Previous studies ${ }^{18,28}$ have reported variations in physical activity and active transportation patterns, respectively, in lowincome versus high-income countries, suggesting that there may be a need for distinct strategies and priorities in different regions or countries. As such, strategies that group countries with similar challenges and/or priorities into clusters by common indicators such as the $\mathrm{HDI}^{29}$ or Gini coefficient ${ }^{29}$ (a measure of income distribution within a population and an indicator of income inequality, where 0 represents perfect income equality [ie, everyone has the same income] and 1 corresponds to perfect income inequality [ie, one person has all the income while everyone else has zero income]), may lead to an improved understanding of the extent of insufficient physical activity among children and youth. Ultimately, this knowledge can be applied to develop strategies and leverage policies to increase opportunities for and access to safe and enjoyable physical activity. This knowledge may also help to identify priority areas for research and surveillance of activity behaviors in countries with low to medium HDIs, which could further support pragmatic and context-specific strategies to promote active lifestyles among children and youth. This study combines report card results from 9 self-selected countries with low to medium HDIs that agreed and registered to participate in the Global Matrix 3.0 initiative. The main objective of this study was to compare the synthesized data on 10 physical activity indicators for children and youth from Bangladesh, Botswana, Ethiopia, Ghana, India, Nepal, Nigeria, South Africa, and Zimbabwe.

\section{Methods}

The 9 participating countries representing low to medium HDIs identified national experts working in various sectors of physical activity and formed country-specific report card working groups that were responsible for rigorous and transparent data and information gathering. Each participating country was assigned an experienced report card mentor, familiar with that country's context. In addition, participating countries received monthly e-blasts from the AHKGA that detailed steps and timelines of the report card development process throughout the year preceding the official launch of the Global Matrix 3.0.
Strategies to gather data included manual or hand searches, online literature searches, and systematic or narrative reviews to obtain the best available peer-reviewed evidence, reporting on the various indicators of physical activity for children and youth ages 5 to 17. In addition, unpublished research (eg, dissertations and theses, government reports) and policy documents were actively sought and gathered through engagement with local stakeholders. These data and information were aggregated and consolidated into report cards following a harmonized process. ${ }^{23,24,26}$

Ten core indicators for the Global Matrix 3.0 (overall physical activity, organized sport and physical activity, active play, active transportation, sedentary behaviors, physical fitness, family and peers, school, community and environment, and government) were used to develop each country-specific report card. For the purposes of analyses, 9 indicators (excluding physical fitness) were grouped into 1 of 2 categories: daily behaviors (overall physical activity, organized sport and physical activity, active play, active transportation, and sedentary behaviors) and settings and sources of influence (family and peers, school, community and environment, and government). Grades ( $\mathrm{A}=$ excellent to $\mathrm{F}=$ failing) were assigned to each indicator using a standardized grading rubric and participating countries adhered to the same benchmarks for grade assignment. ${ }^{25}$ In cases in which data were insufficient to accurately assign a grade, an incomplete was assigned to that indicator.

Although the quality, quantity, and sources of data varied, the report card working groups in each of the 9 countries followed a similar process of appraising, aggregating, consolidating, and harmonizing the total available evidence, discussing discrepancies and reaching consensus before assigning a grade for each indicator. Assigned grades and justifications were submitted to and audited by the scientific subcommittee of the AHKGA. As an example of how indicators were graded, Table 1 presents the methods, instruments, and sample sizes of the studies used to evaluate the overall physical activity indicator. In addition to an abstract and a poster presented at the launch of the Global Matrix 3.0, participating countries consolidated their results into short-form and long-form report cards and were encouraged to develop and publish countryspecific manuscripts based on their findings. Furthermore, participating countries had the latitude to include additional indicator(s) that were specific to their context in their report cards. A companion article ${ }^{25}$ published in this issue of The Journal of Physical Activity and Health provides a detailed description of the methods used by each of the participating countries. In addition, summary papers for each of the participating countries' report cards ${ }^{30-38}$ are included in this issue and provide additional details of data sources.

Descriptive statistics (average grade and SD) were computed after converting categorical variables (letter grades) to interval variables (eg, $\mathrm{A}+=15, \mathrm{~A}=14, \mathrm{~A}-=13, \mathrm{D}=5, \mathrm{D}-=4, \mathrm{~F}=2$ ). Once converted to interval variables, scores for each group of indicators (overall, daily behaviors, settings, and sources of influence) were calculated by summing the relevant interval data. Incomplete grades were removed and the scores were reweighted accordingly. Letter grades (categorical variables) were grouped into 4 levels (A-B, C, D-F, and no grade). The categories were then used to rank countries by letter grade/score and category level in scatter plots. For all correlation analyses among the 10 core indicators and global indices and descriptors (HDI, Gini coefficient, gender inequality, mean years of schooling, public health expenditure, ${ }^{29}$ and improved drinking water coverage ${ }^{39}$ ), Spearman's rank correlation coefficients were calculated. Rule of thumb cutoffs ${ }^{40,41}$ were applied to define weak, moderate, and strong correlations. Pairwise deletion was used to treat missing data 
Table 1 Methods and Instruments Used to Assess Overall Physical Activity

\begin{tabular}{|c|c|c|c|c|}
\hline Country & Method(s) & Instrument used & Age group & Sample size(s) \\
\hline Bangladesh & Subjective & Self-administered questionnaire-GSHS & $13-17$ & 2989 \\
\hline Botswana & Subjective & Literature search, gray literature, and anecdotal information & $9-18$ & N/A \\
\hline Ethiopia & Subjective & Literature, policy documents, and expert interviews & $5-17$ & N/A \\
\hline Ghana & Subjective & $\begin{array}{l}\text { Literature search, focus group interviews on indicators and benchmarks, } \\
\text { policy implementation guidelines, and expert opinions }\end{array}$ & $9-15$ & N/A \\
\hline \multirow[t]{5}{*}{ India } & Objective & Accelerometer & $9-11$ & 1002 \\
\hline & Objective & Accelerometer and self-administered questionnaire & $12-17$ & 324 \\
\hline & Subjective & Self-administered questionnaire & $6-19$ & 27,972 \\
\hline & Subjective & Interviewer-administered questionnaire & $3-15$ & 2734 \\
\hline & Subjective & Interviewer-administered questionnaire & $8-21$ & 325 \\
\hline Nepal & Subjective & Questionnaire & $15-19$ & 241 \\
\hline \multirow[t]{2}{*}{ Nigeria } & Subjective & Researcher-administered semistructured questionnaire & $3-18$ & 1760 \\
\hline & Subjective & Self-administered questionnaire—Health Risk Behavior Questionnaire & $10-21$ & 348 \\
\hline \multirow[t]{4}{*}{ South Africa } & Objective & Accelerometer & $9-11$ & 453 \\
\hline & Subjective & Self-administered questionnaire & $8-14$ & 7348 \\
\hline & Subjective & Self-administered questionnaire & $13-14$ & 239 \\
\hline & Subjective & Self-administered questionnaire & $8-12$ & 832 \\
\hline \multirow[t]{2}{*}{ Zimbabwe } & Subjective & Self-administered questionnaire & $8-16$ & 4402 \\
\hline & Subjective & Self-administered questionnaire-GSHS & $13-15$ & 5665 \\
\hline Total sample(s) & & & & 56,634 \\
\hline
\end{tabular}

Abbreviations: GSHS, global school-based student health survey; N/A, not available.

(incomplete grades). All statistical analyses were performed using $\mathrm{R}$ (version 3.4.1; The R Foundation for Statistical Computing, Vienna, Austria). To extend base R, several packages were loaded, including corrplot, ${ }^{42}$ ggplot $2,{ }^{43}$ UpSetR, ${ }^{44}$ and VIM. ${ }^{45}$

\section{Results}

Table 2 presents the sociodemographic information of the 9 countries with low to medium HDIs participating in the Global Matrix 3.0 initiative (6 sub-Saharan African and 3 Asian countries), with HDIs $^{29}$ ranging from 0.448 (Ethiopia) to 0.698 (Botswana). These countries represent a combined total population of approximately 1.9 billion, with India accounting for 1.3 billion people. ${ }^{46}$ Botswana (4 people $/ \mathrm{km}^{2}$ ) is the least densely populated of the 9 countries, whereas Bangladesh (1252 people $\left./ \mathrm{km}^{2}\right)$ is the most densely populated of the 9 countries. ${ }^{47}$ The physical activity grades for the 10 core indicators, organized by country in alphabetical order, are presented in Table 3. Active transportation is the only core indicator that was assigned a grade by all 9 countries. Physical fitness, which was not a core indicator for the first 2 Global Matrix initiatives, ${ }^{20,21}$ was assigned incomplete grades in all countries except India (grade F). Except for sedentary behaviors (grade range A- to F) and family and peers (grade range A to F), there was a relatively narrow spread in grades for all the indicators (overall physical activity $\mathrm{C}+$ to $\mathrm{D}$, organized sport and physical activity $\mathrm{B}$ to $\mathrm{D}$, active play $\mathrm{B}$ to $\mathrm{D}-$, active transportation $\mathrm{A}-$ to $\mathrm{C}$, school $\mathrm{C}$ to $\mathrm{D}-$, community and environment $\mathrm{C}-$ to $\mathrm{F}$, and government B to D) across the 9 countries.

Grade counts, number of incomplete grades, and mean letter grades for each core indicator are presented in Table 4. In general, the mean grades for daily behaviors were higher $(C)$ than the mean grades for settings and sources of influence (D+), and the overall mean grade for all indicators was a $\mathrm{C}-$. Active transportation and sedentary behaviors had the highest mean grade $(\mathrm{C}+)$, while physical fitness had the lowest $(\mathrm{F})$. Figure 1 provides a comparison of the frequency of letter grades for daily behaviors versus the settings and sources of influence for the 9 countries. The results show that letter grade $\mathrm{C}(19.8 \%)$ was the most frequent for daily behaviors, while INC (13.6\%), closely followed by C and D (12.3\%), were the most frequent grades for settings and sources of influence. Twenty-nine $(32.2 \%)$ of the possible 90 grades were assigned as incomplete. A matrix-based plot of incomplete grades is shown in Figure 2. Ethiopia had only 1 incomplete grade; the other 8 countries had at least 2 or more incomplete grades. Three countries (Bangladesh, Botswana, and Nepal) had at least 5 incomplete grades each. Organized sport and physical activity, physical fitness, and family and peers were graded incomplete in at least 4 countries.

Table 5 presents results of the correlation analyses among the core indicators and some key global indices and descriptors for economic development and health. The findings show mostly weak negative and positive relationships among the global indices (HDI, Gini coefficient, improved drinking water coverage, gender inequality index, public health expenditure, and mean years of schooling) and active transportation, sedentary behaviors, and the school indicators. There were moderate to strong ${ }^{40,41}$ relationships among global indices with overall physical activity, organized sport and physical activity, active play, family, community and environment, and government. The Gini coefficient ${ }^{29}$ had a statistically significant positive $(0.70)$ and negative $(-0.83)$ relationship with overall physical activity and active play, respectively. Public health expenditure ${ }^{29}$ had a statistically significant negative $(-0.87)$ relationship with the community and environment indicator. Mean years of schooling ${ }^{29}$ had statistically significant positive $(0.71)$ and negative $(-0.83)$ relationships with overall physical activity and active play, respectively. Country-specific infant mortality rate ${ }^{48}$ had a statistically significant negative relationship with sedentary 

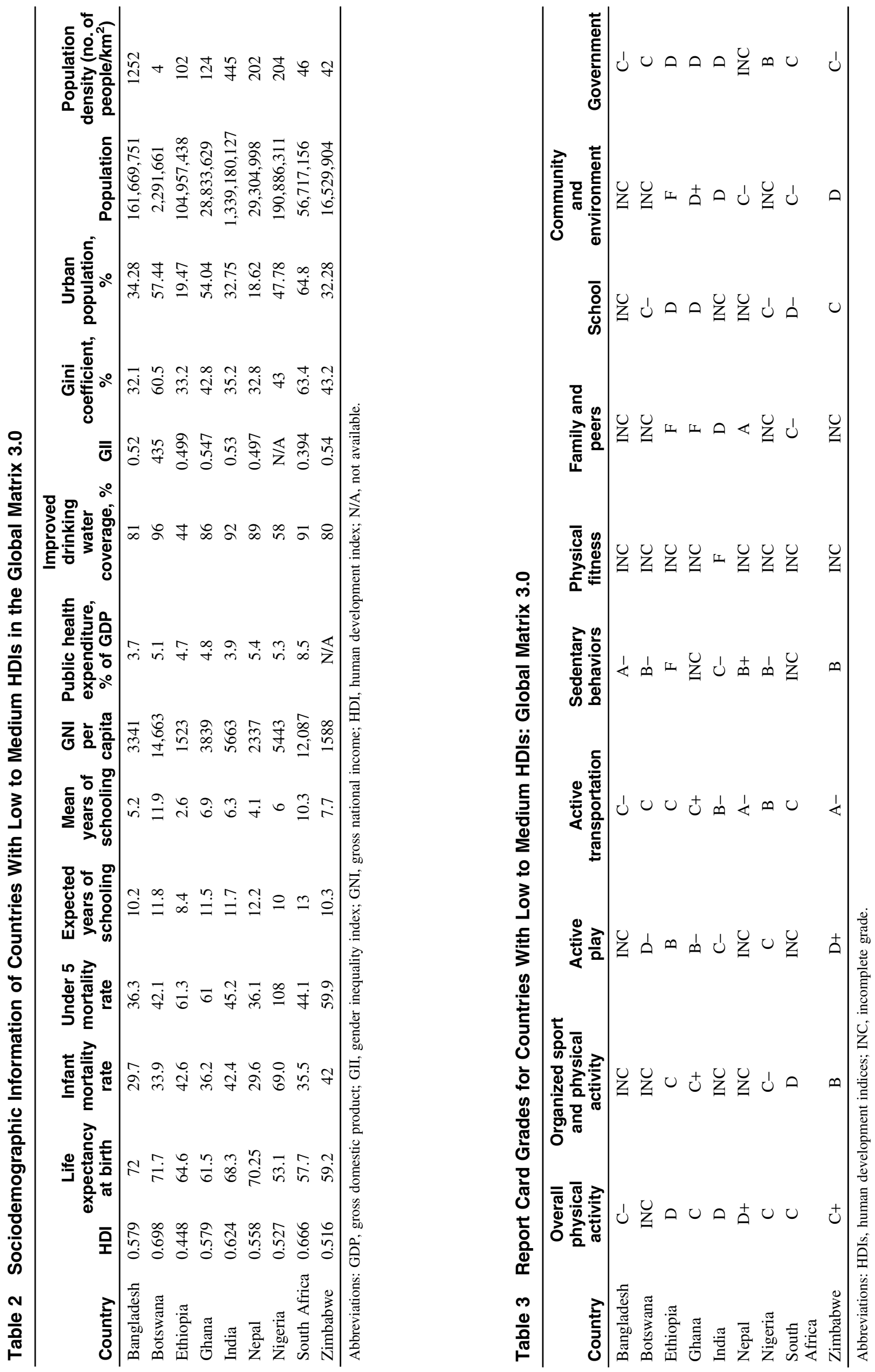
Table 4 Grade Counts and Mean Letter Grades for Core Indicators

\begin{tabular}{|c|c|c|c|c|c|}
\hline Core indicator & Grade count & Incomplete grades & Mean number grade & SD & Mean letter grade \\
\hline Overall physical activity & 8 & 1 & 7 & 1.5 & $\mathrm{C}-$ \\
\hline Organized sport and physical activity & 5 & 4 & 8 & 2.2 & $\mathrm{C}$ \\
\hline Active play & 6 & 3 & 7.7 & 2.6 & $\mathrm{C}-$ \\
\hline Active transportation & 9 & 0 & 9.7 & 2.2 & $\mathrm{C}+$ \\
\hline Sedentary behaviors & 7 & 2 & 9.3 & 3.7 & $\mathrm{C}+$ \\
\hline Physical fitness & 1 & 8 & 2 & NA & $\mathrm{F}$ \\
\hline Family and peers & 5 & 4 & 6 & 4.9 & $\mathrm{D}+$ \\
\hline School & 6 & 3 & 6 & 1.5 & $\mathrm{D}+$ \\
\hline Community and environment & 6 & 3 & 5.3 & 1.9 & $\mathrm{D}$ \\
\hline Government & 8 & 1 & 7 & 2.1 & $\mathrm{C}-$ \\
\hline Behavioral indicators & 9 & 0 & 8.3 & 1.3 & $\mathrm{C}$ \\
\hline Setting and sources of influence indicators & 9 & 0 & 6.7 & 2.1 & $\mathrm{D}+$ \\
\hline All indicators & 9 & 0 & 7.6 & 1.6 & $\mathrm{C}-$ \\
\hline
\end{tabular}

Note: Physical fitness was not included in the behavioral indicators cluster. There are no missing grades for the bottom 3 rows because these scores were adjusted for missing grades.

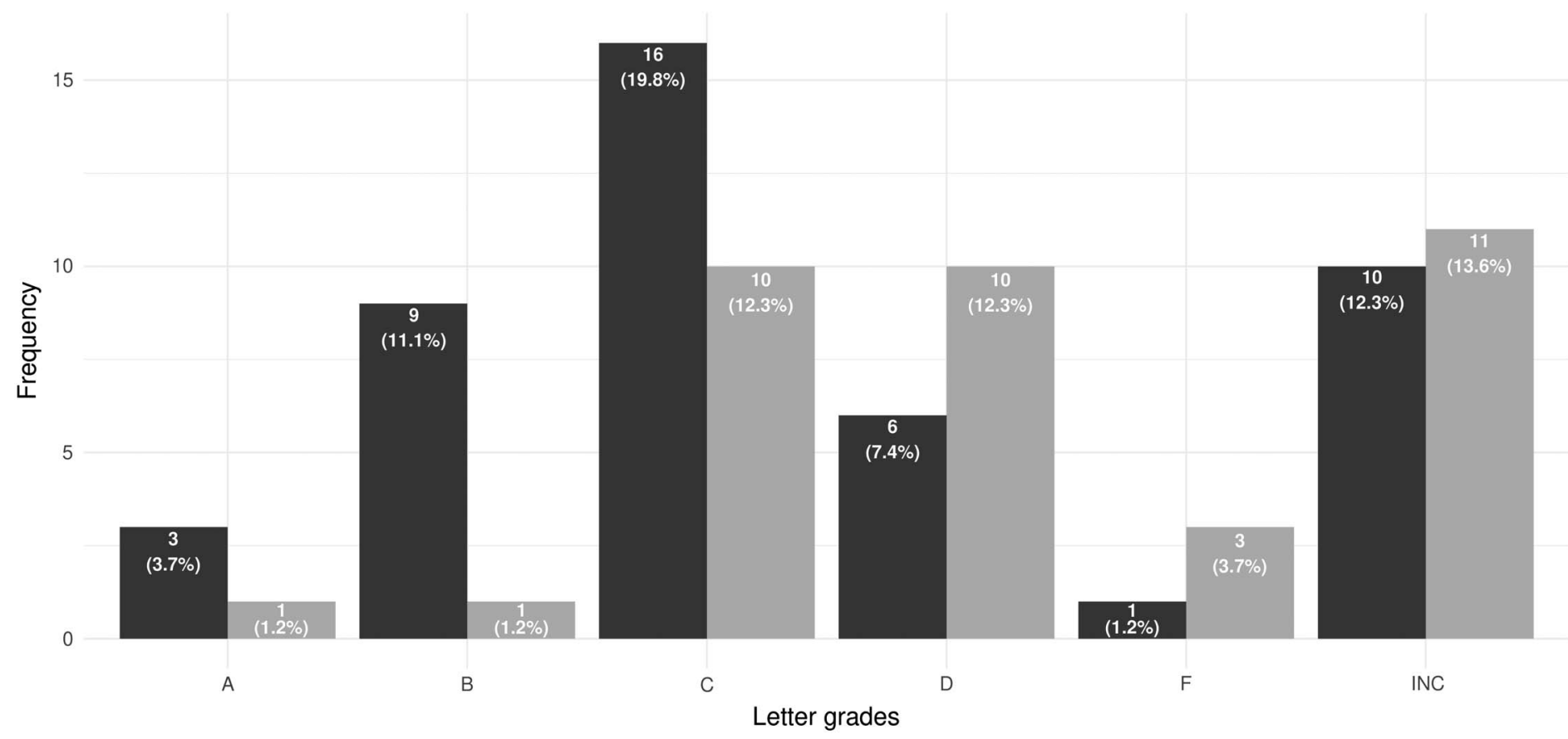

Figure 1 - Frequency of each letter grade for behavioral and settings and sources of influence indicators. INC indicates incomplete grade.

behavior (-0.85). Similarly, the under-five mortality rate ${ }^{48}$ had a statistically significant negative relationship with the family and peers indicator $(-0.97)$.

\section{Discussion}

Findings from this study provide a broad examination of grades assigned to 10 indicators of physical activity among children and youth from 9 countries with low to medium HDIs that participated in the Global Matrix 3.0 initiative. ${ }^{25}$ Although significant effort was made to standardize and gather the best country-specific data, our findings are limited to the overall quality of data and the number of indicators that were examined and assigned grades. As such, caution is needed in interpreting these results. Although the 9 countries included in this study are spread across Africa and Asia, with diverse cultural and geographical contexts within and between them, the narrow spread and similar clustering of grades for most indicators (Table 3 ) suggest that these countries have comparable challenges, such as a surge in inactive lifestyles among children and youth. The findings point toward a need for increased 

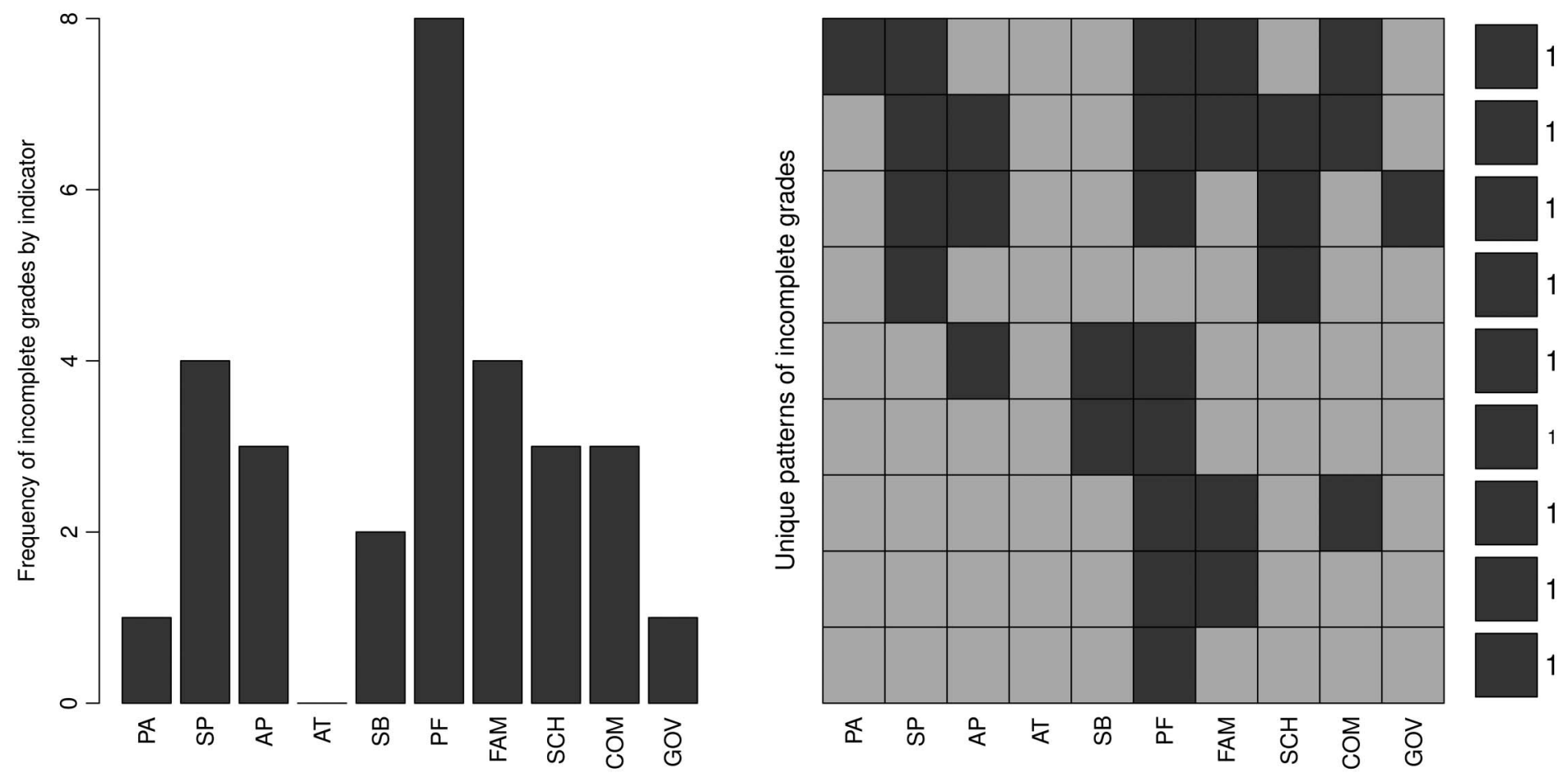

Figure 2 - Matrix-based plot of incomplete grades. Note: In the plot to the right of the figure, dark gray cells represent missing grades. AP indicates active play; AT, active transportation; COM, community and environment; FAM, family and peers; GOV, government; PA, physical activity; PF, physical fitness; SB, sedentary behaviors; SCH, school; SP, organized sport and physical activity.

Table 5 Correlation Matrix (Spearman's Rank Correlation Coefficients) of Mean Physical Activity Grades by Global Indices and Descriptors

\begin{tabular}{|c|c|c|c|c|c|c|c|c|c|}
\hline \multirow[b]{2}{*}{ Sociodemographic index/descriptor } & \multicolumn{9}{|c|}{ Core indicators } \\
\hline & PA & SP & AP & AT & SB & FAM & $\mathrm{SCH}$ & COM & GOV \\
\hline Human development index ${ }^{29}$ & -0.02 & -0.5 & -0.6 & -0.41 & 0.18 & 0.31 & -0.21 & 0.62 & 0.29 \\
\hline Gini coefficient ${ }^{29}$ & 0.70 & -0.3 & -0.83 & 0.06 & -0.36 & 0.21 & 0 & 0.18 & 0.62 \\
\hline Improved drinking water coverage ${ }^{39}$ & -0.16 & -0.2 & -0.71 & -0.11 & 0.20 & 0.56 & -0.12 & 0.53 & 0.06 \\
\hline Gender inequality index ${ }^{29}$ & 0.29 & 0.8 & 0.3 & 0.37 & 0.03 & -0.72 & 0.41 & -0.44 & -0.66 \\
\hline Public health expenditure ${ }^{29}$ & 0.51 & -0.8 & -0.2 & 0.41 & 0.06 & 0.56 & -0.11 & 0.87 & 0.67 \\
\hline Mean years of schooling 29 & 0.71 & -0.1 & -0.83 & -0.06 & 0.02 & 0.15 & 0.09 & 0.44 & 0.38 \\
\hline Infant mortality rate ${ }^{48}$ & -0.03 & -0.3 & 0.54 & -0.14 & -0.85 & -0.21 & -0.59 & -0.26 & -0.29 \\
\hline Under 5 mortality rate ${ }^{48}$ & 0.18 & 0 & 0.77 & 0.16 & -0.65 & -0.97 & 0.02 & -0.79 & -0.11 \\
\hline Urban population ${ }^{49}$ & 0.49 & -0.5 & -0.43 & -0.48 & -0.02 & -0.21 & -0.41 & 0.26 & 0.56 \\
\hline
\end{tabular}

Abbreviations: AP, active play; AT, active transportation; COM, community and environment; FAM, family and peers; GOV, government; PA, physical activity; $\mathrm{SB}$, sedentary behaviors; SCH, school; SP, organized sport and physical activity. Note: Correlation coefficients in bold show statistically significant $(P<.05)$ moderate to very strong ${ }^{40,41}$ (negative or positive) relationships between a specific indicator and an index/descriptor. Physical fitness was removed from this analysis due to a majority (8/9) of incomplete grades. Pairwise deletion was used to treat missing data.

knowledge exchange and collaboration between countries to develop common goals and strategies to address insufficient physical activity and increased sedentary behaviors among children and youth. This observation is in line with a recommendation made in the Lancet physical activity series ${ }^{50}$ to promote capacity building, workforce training, and intersectoral approaches for physical activity research.

Active transportation was the only indicator assigned a grade by all 9 countries. In resource-limited countries, focusing on the promotion and preservation of behaviors such as active transportation may be the most practical and achievable means to improve physical activity among children and youth. In most of the participating countries, active transportation is still a necessary part of life for most children and youth. The argument for promoting active transportation is supported by the observation that, in general, grades for daily physical activity behaviors were higher than those for settings and sources of influence. Promoting the benefits of active transportation should be weighed against the unintended safety risks of potential harm to pedestrians and cyclists, especially in less walkable urban areas. ${ }^{7}$ Although strong and well-designed settings and sources of influence may be preferred, especially given their reported influence on children's participation 
in physical activity, ${ }^{51}$ the lack of resources, political will, ${ }^{52}$ as well as competing priorities, are a reality that cannot be easily addressed in most countries with low-to-medium HDIs. Moreover, having strong and supportive settings and sources of influence does not guarantee increased physical activity participation, as demonstrated by results from the Global Matrix 2.0, ${ }^{23}$ which showed lower levels of overall physical activity among children and youth from countries such as Belgium, ${ }^{53}$ Sweden, ${ }^{54}$ and Denmark, ${ }^{55}$ which have very supportive infrastructure.

Findings from the analyses of this study also demonstrate a dearth of data in the participating countries, which has been previously reported ${ }^{7,56}$ for most of the indicators, and we present results that are mainly descriptive and correlational; thus, interpretations need to be made with caution. The information in Table 1 presents the common, objective data limitations that are unique to most countries with low to medium HDIs. ${ }^{1,27}$ Except for South Africa and India ( 2 medium HDI countries with some accelerometer data), the other 7 countries relied on subjective data and/or expert opinions to evaluate overall physical activity. Such reliance on subjective data, which are known to be prone to recall and other biases, ${ }^{57}$ may impact the accuracy of the reported results. Furthermore, over one-third of the indicators could not be assigned grades due to the unavailability or insufficiency of relevant data. Such data gaps and the preponderant reliance on subjective measures demands urgent action to invest in objective research in countries with low to medium HDIs. ${ }^{27}$ This would be a critical step in providing empirical evidence in order to facilitate not only consistent global comparisons and accurate trends over time but also to inform evidence-based policies. The need to prioritize and strengthen systematic surveillance of the core indicators of physical activity among children and youth in countries with low to medium HDIs is further demonstrated by the fact that 8 out of the 9 participating countries could not assign a grade for physical fitness, a simple and cost-effective assessment, ${ }^{58}$ due to lack of data.

Accurately interpreting the findings from the present correlation analyses is challenging because of the potential influence of many mechanisms and/or factors that may confound the relationships. A study ${ }^{59}$ among American adults found that state-level income inequality was associated with increased risk of sedentary behavior. Whether or not these findings are generalizable to other countries with low to medium HDIs requires further investigation. Mechanisms through which income inequality may affect overall physical activity or sedentary behavior include its relationship with depression, ${ }^{60,61}$ which in turn is reportedly associated with decreased participation in physical activity ${ }^{60}$ and increased sedentary behavior among adolescents ${ }^{62,63}$ and adults. ${ }^{59}$ Income inequality has also been found to be associated with depressive symptoms among adolescent girls. ${ }^{64}$ Similar to the Global Matrix 2.0 findings, ${ }^{23}$ a recent study ${ }^{65}$ found that an increased Gini coefficient was associated with decreased participation in physical activity. Another study of 76 countries $^{20}$ showed a positive association between physical inactivity and HDI (ie, countries' physical inactivity increased in tandem with an increasing HDI). Contrary to Dumith et al's ${ }^{20}$ findings, a study of 47 countries (mostly low-middle-income countries), ${ }^{66}$ reported an inverse relationship between HDI and physical inactivity, indicating that as human development increased, physical inactivity decreased. Findings from this study show a negligible $(-0.02)$ relationship between HDI and grades for overall physical activity, suggesting that there is no relationship between the two. The negligible relationship found in this study may be due to the narrow variance in the HDIs of the participating countries, deliberately selected to create a more homogenous sample. These conflicting results may also be due to differences in the composition of samples, highlighting the possible need to further explore these relationships using larger and more diverse samples of countries.

The significantly positive relationships between grades for overall physical activity and the Gini coefficient, overall physical activity and mean years of schooling, and community and environment with public health expenditure, and the significantly negative relationships between active play and Gini coefficient, and active play and mean years of schooling need to be interpreted with caution. First, the sample of countries included in this study $(n=9)$ is very small. Second, missing data were treated using pairwise deletion under the missing at random assumption. Third, data to compute the Gini coefficient, the mean years of schooling, and public health expenditure, ${ }^{29}$ were from 2016, while countryspecific data and evidence used to assign grades may have been obtained from different years. Fourth, the present correlation analyses did not account for potential confounders. Furthermore, the quality, sources, and quantity of the data informing the grades varied from country to country. Active play among children and youth can be culture and context specific; given the inevitable cultural and contextual variability among the included countries, the significant correlational results should be interpreted with caution. In addition, given the reported relationships between income inequality and sedentary behavior ${ }^{59}$ and physical activity, ${ }^{65}$ the negligible to weak relationships observed among sedentary behaviors and the indices and descriptors may be spurious. Alternatively, these findings may suggest that recreational screen time is low in countries with low to medium HDIs, or that the weak relationships observed may be emblematic of the weaknesses in the measurement of sedentary behaviors.

Some studies from high-income countries have reported inverse relationships between income inequality and general health, ${ }^{67,68}$ physical activity, ${ }^{69}$ and leisure-time physical activities. ${ }^{70}$ Furthermore, Tomkinson et al $^{71}$ reported a strong inverse relationship between cardiorespiratory fitness and the Gini coefficient in 18 mostly high-income countries. However, care should be taken not to overgeneralize based on these findings given the need for research to determine if such results can replicated using samples from countries with low-medium HDIs. The strong positive relationship between the Gini coefficient and overall physical activity in the present analyses is in contrast to findings by Elgar et $\mathrm{al}^{69}$ and the Global Matrix 2.0, ${ }^{23}$ which showed negative relationships between these two, indicating that higher national income inequality is related to less physical activity among adolescents. The explanation for these differences may lie in differences in measurement methodologies or contexts. Alternatively, our findings may be subject to the ecological fallacy (ie, we cannot attribute findings observed at the population level to an individual). ${ }^{20}$ However, our findings might also reflect the substantial needs-based physical activity ${ }^{72}$ (eg, household chores, farming, and active transportation) among the low-income families who are the majority in countries with low to medium HDIs; in such environments, opportunities for active play may be few, deemed a luxury, and may possibly be unsafe.

Higher grades for daily behaviors for countries with low to medium HDIs participating in the Global Matrix 3.0 is comparable with results for low- to medium-income countries ${ }^{73-78}$ that participated in the 2016 Global Matrix 2.0 initiative. ${ }^{23}$ These findings demonstrate that, although there may be ongoing physical activity transitions ${ }^{8,19}$ in countries with low to medium HDIs, there may still be an opportunity to reduce the transition acceleration. To this 
end, it may be reasonable to employ the common global $^{79,80}$ and/or regional $^{81}$ strategies that have been proposed and other recommendations, ${ }^{50,56}$ including those for improving grades from initiatives such as the Global Matrix, ${ }^{23-25}$ in the fight against insufficient physical activity among children and youth. Perhaps it is time to capitalize on the momentum created by such a collective search for practical and realistic solutions. Therefore, investing resources in initiatives such as the Global Matrix ${ }^{23,24}$ and the recent push for 24-hour movement guidelines for the early years $, 82,83$ including those currently being developed by the World Health Organization, the United Kingdom, and South Africa, ${ }^{27}$ should be vigorously pursued. For example, organizations such as the African Physical Activity Network ${ }^{81}$ and the AHKGA, must be empowered to act either as conduits or as the primary drivers of collaborative efforts among academics, researchers, and policymakers in their respective regions.

\section{Strengths and Limitations}

The strengths of this study include providing the most comprehensive assessment of physical activity levels among children and youth in the 9 participating countries with low to medium HDIs. Grade assignments for the core indicators were informed by the best available data from each of the participating countries, gathered and synthesized using a transparent and harmonized approach. The Global Matrix 3.0 initiative and the development of this study provided an opportunity for cross-fertilization of ideas from academics, researchers, and policymakers alike, and the possibility to generate common strategies to address the insufficient physical activity epidemic among children and youth in countries with low to medium HDIs. However, there are some key limitations that are important to mention. The quality and quantity of data varied significantly across the countries. Due to insufficient data, many indicators could not be assigned grades, which limits the comparisons and inferences that can be made. The composition and expertise of the country-specific report card working groups varied. This study only includes data from 9 countries with low to medium HDIs, and as such, may not be representative of all other countries with similar challenges and opportunities.

\section{Conclusions}

This study provides a comprehensive assessment of physical activity and related behaviors among children and youth and the settings and sources of influence in 9 countries with low to medium HDIs participating in the Global Matrix 3.0 initiative. The study provides clear evidence that there is a need for high quality, nationally representative data at the country and regional level in order to better understand the levels of physical activity in countries with low to medium HDIs. Findings from the present analyses show that most of the 9 participating countries had higher grades for behavioral indicators despite having lower grades for indicators of settings and sources of influence, suggesting that favorable community environments may not be the only driver of increased physical activity levels. Findings also show that, in general, levels of physical activity among children and youth in the 9 countries with low to medium HDIs are lower than desired. The lack of objective data and the overall low quality of the data among the participating countries presents collaborative research opportunities for the AHKGA and other organizations to develop strategies to collectively address the existing data gaps.

\section{Acknowledgments}

The authors would like to acknowledge the Active Healthy Kids Canada (now ParticipACTION) for developing the report card methodology and the AHKGA for modifying and standardizing the benchmarks and grading rubric. The authors are grateful for all the hard work by each participating country's report card working group and all other members of their report card committees. T.M. is supported by a Canadian Institute of Health Research (CIHR) fellowship.

\section{References}

1. Sallis JF, Bull F, Guthold R, et al. Progress in physical activity over the Olympic quadrennium. Lancet. 2016;388(10051):1325-1336. PubMed ID: 27475270 doi:10.1016/S0140-6736(16)30581-5

2. Janssen I, Leblanc AG. Systematic review of the health benefits of physical activity and fitness in school-aged children and youth. Int $J$ Behav Nutr Phys Act. 2010;7:40. PubMed ID: 20459784 doi:10. 1186/1479-5868-7-40

3. Poitras VJ, Gray CE, Borghese MM, et al. Systematic review of the relationships between objectively measured physical activity and health indicators in school-aged children and youth. Appl Physiol Nutr Metab. 2016;41(6, suppl 3):S197-S239. PubMed ID: 27306431 doi:10.1139/apnm-2015-0663

4. Donnelly JE, Lambourne K. Classroom-based physical activity, cognition, and academic achievement. Prev Med. 2011;52(suppl 1): S36-S42. doi:10.1016/j.ypmed.2011.01.021

5. Hillman CH, Pontifex MB, Raine LB, Castelli DM, Eric E, Kramer AF. Control and academic achievement in preadolescent. Children. 2010;159(3):1044-1054. doi:10.1016/j.neuroscience.2009.01.057.THE

6. Rhodes RE, Janssen I, Bredin SSD, Warburton DER, Bauman A. Physical activity: health impact, prevalence, correlates and interventions. Psychol Health. 2017;32(8):942-975. PubMed ID: 28554222 doi:10.1080/08870446.2017.1325486

7. Hallal PC, Andersen LB, Bull FC, et al. Global physical activity levels: surveillance progress, pitfalls, and prospects. Lancet. 2012;380(9838):247-257. PubMed ID: 22818937 doi:10.1016/ S0140-6736(12)60646-1

8. Muthuri SK, Wachira LJM, Leblanc AG, et al. Temporal trends and correlates of physical activity, sedentary behaviour, and physical fitness among school-aged children in Sub-Saharan Africa: a systematic review. Int J Environ Res Public Health. 2014;11(3):3327-3359. PubMed ID: 24658411 doi:10.3390/ijerph110303327

9. World Health Organization (WHO). Report of the Commission on Ending Childhood Obesity. Geneva, Switzerland: World Health Organization; 2016:30. ISBN: 9789241510066

10. Tremblay MS, LeBlanc AG, Kho ME, et al. Systematic review of sedentary behaviour and health indicators in school-aged children and youth. Int J Behav Nutr Phys Act. 2011;8(1):98. doi:10.1186/14795868-8-98

11. Carson V, Janssen I. Volume, patterns, and types of sedentary behavior and cardio-metabolic health in children and adolescents: a cross-sectional study. BMC Public Health. 2011;11(1):274. doi:10. 1186/1471-2458-11-274

12. Carson V, Chaput JP, Janssen I, Tremblay MS. Health associations with meeting new 24-hour movement guidelines for Canadian children and youth. Prev Med. 2017;95:7-13. doi:10.1016/j.ypmed.2016. 12.005

13. González K, Fuentes J, Márquez JL. Physical inactivity, sedentary behavior and chronic diseases. Korean J Fam Med. 2017;38(3):111115. doi:10.4082/kjfm.2017.38.3.111 
14. World Health Organisation. Global Recommendations on Physical Activity for Health. Geneva, Switzerland: World Health Organisation; 2011. doi:10.1080/11026480410034349

15. United Nations Department of Economic Development. World Urbanization Prospects 2018- Urbanization around the world Multimedia Library_-United Nations Department of Economic and Social Affairs. 2018. https://www.un.org/development/desa/publications/ graphic/world-urbanization-prospects-2018-urbanization-around-theworld. Accessed June 30, 2018.

16. Johnson-Lawrence V, Schulz AJ, Zenk SN, et al. Joint associations of residential density and neighborhood involvement with physical activity among a multiethnic sample of urban adults. Health Educ Behav. 2015;42(4):510-517. doi:10.1177/1090198114564500

17. Guthold R, Ono T, Strong KL, Chatterji S, Morabia A. Worldwide variability in physical inactivity. Am J Prev Med. 2008;34(6):486494. PubMed ID: 18471584 doi:10.1016/j.amepre.2008.02.013

18. Larouche R, Sarmiento OL, Broyles ST, et al. Are the correlates of active school transport context-specific? Int J Obes Suppl. 2015;5: S89-S99. PubMed ID: 27152191 doi:10.1038/ijosup.2015.25

19. Katzmarzyk PT, Mason C. The physical activity transition. J Phys Act Health. 2009;6(1):269-280. doi:10.1123/jpah.6.3.269

20. Dumith SC, Hallal PC, Reis RS, Kohl HW. Worldwide prevalence of physical inactivity and its association with human development index in 76 countries. Prev Med. 2011;53(1-2):24-28. doi:10.1016/j. ypmed.2011.02.017

21. United Nations. Ensure healthy lives and promote well being for all at all ages. 2015. https://una-gp.org/clancyt/files/goals/goal3.pdf. Accessed June 30, 2018.

22. World Health Organization. Draft WHO global action plan on physical activity 2018-2030 INTRODUCTION Background. 2017. http:// www.who.int/ncds/governance/gappa_version_4August2017.pdf. Accessed June 30, 2018.

23. Tremblay MS, Barnes JD, González SA, et al. Global matrix 2.0: report card grades on the physical activity of children and youth comparing 38 countries and the global matrix 2.0 research team. $J$ Phys Act Health. 2016;13(suppl 2):S343-S366. doi:10.1123/jpah. 2016-0594

24. Tremblay MS, Gray CE, Akinroye K, et al. Physical activity of children: a global matrix of grades comparing 15 countries. J Phys Act Health. 2014;11(suppl 1):S113-S125. doi:10.1123/jpah.2014-0177

25. Aubert S, Barnes J, Abdeta C, et al. Global matrix 3.0 physical activity report card grades for children and youth: results and analysis from 49 countries. J Phys Act Health. 2018;15(suppl 2):S251-S273. doi:10.1123/jpah.2018-0472

26. Colley RC, Brownrigg M, Tremblay MS. A model of knowledge translation in health: the Active Healthy Kids Canada Report Card on physical activity for children and youth. Health Promot Pract. 2012;13(3): 320-330. PubMed ID: 22447666 doi:10.1177/1524839911432929

27. Okely AD, Tremblay MS, Reilly JJ, Draper CE, Bull F. Physical activity, sedentary behaviour, and sleep: movement behaviours in early life. Lancet Child Adolesc Health. 2018;2(4):233-235. doi:10. 1016/S2352-4642(18)30070-1

28. Chaput JP, Katzmarzyk PT, LeBlanc AG, et al. Associations between sleep patterns and lifestyle behaviors in children: an international comparison. Int J Obes Suppl. 2015;5(suppl 2):S59-S65. doi:10. 1038/ijosup.2015.21

29. United Nations Development Programme. Human Development Report 2016. New York, NY: United Nations Development Programme; 2016. eISBN: 978-92-1-060036-1

30. Khan A, Kadir MA, Choudhury SR, et al. Results from Bangladesh's 2018 report card on physical activity for children and youth. J Phys Act Health. 2018;15(suppl 2):S318-S319. doi:10.1123/jpah.2018-0419
31. Tladi DM, Monnaatsie M, Shaibu S, et al. Results from Botswana's 2018 report card on physical activity for children and youth. J Phys Act Health. 2018;15(suppl 2):S320-S322. doi:10.1123/jpah.2018-0420

32. Abdeta C, Teklemariam Z, Deksisa A, Abera E. Results from Ethiopia's 2018 report card on physical activity for children and youth. J Phys Act Health. 2018;15(suppl 2):S353-S354. doi:10.1123/ jpah.2018-0457

33. Nyawornota VK, Luguterah A, Sofo S, et al. Results from Ghana's 2018 report card on physical activity for children and youth. J Phys Act Health. 2018;15(suppl 2):S366-S367. doi:10.1123/jpah.2018-0459

34. Bhawra J, Chopra P, Harish R, et al. Results from India's 2018 report card on physical activity for children and youth. J Phys Act Health. 2018;15(suppl 2):S373-S374. doi:10.1123/jpah.2018-0475

35. Subedi N, Paudel S, Nepal S, Karki A, Magar M, Suresh M. Results from Nepal's 2018 report card on physical activity for children and youth. J Phys Act Health. 2018;15(suppl 2):S386-S387. doi:10.1123/ jpah.2018-0512

36. Akinroye KK, Adeniyi AF. Results from Nigeria's 2018 report card on physical activity for children and youth. J Phys Act Health. 2018;15(suppl 2):S393-S394. doi:10.1123/jpah.2018-0514

37. Draper CE, Tomaz SA, Bassett SH, et al. Results from South Africa's 2018 report card on physical activity for children and youth. J Phys Act Health. 2018;15(suppl 2):S406-S408. doi:10.1123/jpah.2018-0517

38. Manyanga T, Munambah NE, Mahachi CB, et al. Results from Zimbabwe's 2018 report card on physical activity for children and youth. J Phys Act Health. 2018;15(suppl 2):S433-S435. doi:10.1123/ jpah.2018-0520

39. United Nations Statistics Division. Social indicators. 2015. https:// unstats.un.org/unsd/demographic/products/socind/.

40. Overholser BR, Sowinski KM. Biostatistics primer: part 2. Nutr Clin Pract. 2008;23(1):76-84. 2008:76-84. PubMed ID: 18203967 doi:10.1177/011542650802300176

41. Schober P, Boer C, Schwarte LA. Correlation coefficients. Anesth Analg. 2018;126(5):1763-1768. doi:10.1213/ANE.0000000000002864

42. Wei T, Simko V, Levy M, Xie Y, Jin YZJ. Visualization of a correlation matrix: corrplot. 2017. https://github.com/taiyun/corrplot.

43. Wickham H. Elegant graphics for data analysis. 2009. https://www. springer.com/gp/book/9780387981413.

44. Conway JR, Lex A, Gehlenborg N. UpSetR: an R package for the visualization of intersecting sets and their properties. Bioinformatics. 2017;33(18):2938-2940. PubMed ID: 28645171 doi:10.1093/ bioinformatics/btx364

45. Kowarik A, Templ M. Imputation with the R Package VIM. J Stat Softw. 2016;74(7):1-16. doi:10.18637/jss.v074.i07

46. United Nations. World population prospects 2017. 2017. https://esa. un.org/unpd/wpp/.

47. The World Bank. Population density (2016). 2016. https://data. worldbank.org/indicator/EN.POP.DNST.

48. World Health Organization. Global Health Observatory country views. http://apps.who.int/gho/data/node.country. Accessed September 8, 2018.

49. World Bank. Urban population Data. https://data.worldbank.org/ indicator/SP.URB.TOTL. Accessed September 11, 2018.

50. Kohl HW, Craig CL, Lambert EV, et al. The pandemic of physical inactivity: global action for public health. Lancet. 2012;380(9838):294305. PubMed ID: 22818941 doi:10.1016/S0140-6736(12)60898-8

51. Davison K, Lawson CT. Do attributes in the physical environment influence children's physical activity? A review of the literature. Int $J$ Behav Nutr Phys Act. 2006;3(1):19. doi:10.1186/1479-5868-3-19

52 . Mendis S. The policy agenda for prevention and control of noncommunicable diseases. Br Med Bull. 2010;96(1):23-43. doi:10. 1093/bmb/ldq037 
53. Wijtzes AI, Verloigne M, Mouton A, et al. Results From Belgium's 2016 report card on physical activity for children and youth. $J$ Phys Act Health. 2016;13(11, suppl 2):S95-S103. doi:10.1123/jpah.2016-0306

54. Nyström CD, Larsson C, Ehrenblad B, et al. Results From Sweden's 2016 report card on physical activity for children and youth. $J$ Phys Act Health. 2016;13(11, suppl 2):S284-S290. doi:10.1123/jpah.2016-0307

55. Larsen LR, Troelsen J, Kirkegaard KL, et al. Results From Denmark's 2016 report card on physical activity for children and youth. $J$ Phys Act Health. 2016;13(11, suppl 2):S137-S142. doi:10.1123/jpah.2016-0403

56. Bauman AE, Reis RS, Sallis JF, Wells JC, Loos RJF, Martin BW. Correlates of physical activity: why are some people physically active and others not? Lancet. 2012;380(9838):258-271. PubMed ID: 22818938 doi:10.1016/S0140-6736(12)60735-1

57. Urda JL, Larouere B, Verba SD, Lynn JS. Comparison of subjective and objective measures of office workers' sedentary time. Prev Med Rep. 2017;8:163-168. PubMed ID: 29062680 doi:10.1016/j.pmedr. 2017.10.004

58. Lang JJ, Tremblay MS, Léger L, Olds T, Tomkinson GR. International variability in $20 \mathrm{~m}$ shuttle run performance in children and youth: who are the fittest from a 50-country comparison? A systematic literature review with pooling of aggregate results. Br J Sports Med. 2018;52(4):276. PubMed ID: 27650256 doi:10.1136/bjsports2016-096224

59. Diez-Roux A V, Link BG, Northridge ME. A multilevel analysis of income inequality and cardiovascular disease risk factors. Soc Sci Med. 2000;50(5):673-687.

60. Ahern J, Galea S. Social context and depression after a disaster: the role of income inequality. $J$ Epidemiol Community Health. 2006; 60(9):766-770. PubMed ID: 16905720 doi:10.1136/jech.2006.042069

61. Pabayo R, Kawachi I, Gilman SE. Income inequality among American states and the incidence of major depression. J Epidemiol Community Health. 2014;68(2):110-115. PubMed ID: 24064745 doi:10.1136/jech-2013-203093

62. Vancampfort D, Stubbs B, Firth J, Van Damme T, Koyanagi A. Sedentary behavior and depressive symptoms among 67, 077 adolescents aged 12-15 years from 30 low- and middle-income countries. Int J Behav Nutr Phys Act. 2018;15(1):73. PubMed ID: 30089487 doi:10.1186/s12966-018-0708-y

63. Maras D, Flament MF, Murray M, et al. Screen time is associated with depression and anxiety in Canadian youth. Prev Med. 2015;73: 133-138. PubMed ID: 25657166 doi:10.1016/j.ypmed.2015.01.029

64. Pabayo R, Dunn EC, Gilman SE, Kawachi I, Molnar BE. Income inequality within urban settings and depressive symptoms among adolescents. J Epidemiol Community Health. 2016;70(10):997-1003. PubMed ID: 27103664 doi:10.1136/jech-2015-206613

65. Pabayo R, Fuller D, Lee EY, Horino M, Kawachi I. State-level income inequality and meeting physical activity guidelines; differential associations among US men and women. J Public Health (Bangkok). 2018;40(2):229-236. doi:10.1093/pubmed/fdx082

66. Atkinson K, Lowe S, Moore S. Human development, occupational structure and physical inactivity among 47 low and middle income countries. Prev Med Rep. 2015;3:40-45. doi:10.1016/j.pmedr.2015. 11.009

67. Van Deurzen I, Van Oorschot W, Van Ingen E. The link between inequality and population health in low and middle income countries: policy myth or social reality? PLoS ONE. 2014;9(12):e115109-22. doi:10.1371/journal.pone.0115109

68. Wilkinson RG, Pickett KE. Income inequality and population health: a review and explanation of the evidence. Soc Sci Med. 2006;62(7): 1768-1784. PubMed ID: 16226363 doi:10.1016/j.socscimed.2005. 08.036
69. Elgar FJ, Pförtner TK, Moor I, De Clercq B, Stevens GW, Currie C. Socioeconomic inequalities in adolescent health 2002-2010: a time-series analysis of 34 countries participating in the Health Behaviour in School-aged Children study. Lancet. 2015;385(9982):2088-2095. PubMed ID: 25659283 doi:10.1016/S0140-6736(14)61460-4

70. Gonzalo-Almorox E, Urbanos-Garrido RM. Decomposing socioeconomic inequalities in leisure-time physical inactivity: the case of Spanish children. Int J Equity Health. 2016;15(1):106. doi:10. 1186/s12939-016-0394-9

71. Tomkinson GR, Lang JJ, Tremblay MS. Temporal trends in the cardiorespiratory fitness of children and adolescents representing 19 high-income and upper middle-income countries between 1981 and 2014 [published online ahead of print October 30, 2017]. Br J Sports Med. doi:10.1136/bjsports-2017-097982

72. Proctor MH, Moore LL, Singer MR, Hood MY, Nguyen US, Ellison RC. Risk profiles for non-communicable diseases in rural and urban schoolchildren in the Republic of Cameroon. Ethn Dis. 1996;6(3-4): 235-243.

73. Onywera VO, Muthuri SK, Hayker S, et al. Results From Kenya's 2016 report card on physical activity for children and youth. $J$ Phys Act Health. 2016;13(11, suppl 2):S195-S200. doi:10.1123/jpah. 2016-0359

74. Prista A, Daca T, Tchonga F, Machava E, Macucule C, Ribeiro E. Results from the Mozambique 2016 report card on physical activity for children and adolescents. J Phys Act Health. 2016;13(11 suppl 2): S213-S217. doi:10.1123/jpah.2016-0526

75. Manyanga T, Makaza D, Mahachi C, et al. Results From Zimbabwe's 2016 report card on physical activity for children and youth. J Phys Act Health. 2016;13(11, suppl 2):S337-S342. doi:10.1123/jpah.20160304

76. Adeniyi AF, Odukoya OO, Oyeyemi AL, et al. Results From Nigeria's 2016 report card on physical activity for children and youth. J Phys Act Health. 2016;13(11, suppl 2):S231-S236. doi: 10.1123/jpah.2016-0305

77. Uys M, Bassett S, Draper CE, et al. Results From South Africa's 2016 report card on physical activity for children and youth. $J$ Phys Act Health. 2016;13(11, suppl 2):S265-S273. doi:10.1123/jpah.20160409

78. Katapally TR, Goenka S, Bhawra J, et al. Results From India's 2016 report card on physical activity for children and youth. $J$ Phys Act Health. 2016;13(11, suppl 2):S176-S182. doi:10.1123/jpah.20160393

79. World Health Organization. Draft WHO Global Action Plan on Physical Activity 2018-2030. Geneva, Switzerland: World Health Organization; Vol 2011. 2017.

80. ISPAH International Society for Physical Activity and Health. The Bangkok declaration on physical activity for global health and sustainable development. Br J Sports Med. 2017;51(19):1389-1391. doi:10.1136/bjsports-2017-098063

81. African Physical Activity Network. The African Physical Activity Declaration. 2016. http://www.icsspe.org/content/african-physicalactivity-declaration.

82. Tremblay MS, Chaput JP, Adamo KB, et al. Canadian 24-hour movement guidelines for the early years ( $0-4$ years): an integration of physical activity, sedentary behaviour, and sleep. BMC Public Health. 2017;17(suppl 5). doi:10.1186/s12889-017-4859-6

83. Okely AD, Ghersi D, Hesketh KD, et al. A collaborative approach to adopting/adapting guidelines-The Australian 24-Hour Movement Guidelines for the early years (Birth to 5 years): an integration of physical activity, sedentary behavior, and sleep. BMC Public Health. 2017;17(suppl 5):869. doi:10.1186/s12889-017-4867-6 